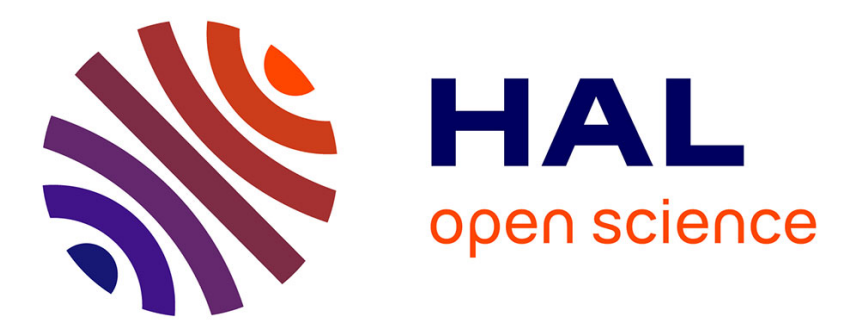

\title{
Chromosome dynamics during interphase: a biophysical perspective
}

\author{
Maxime Mc Tortora, Hossein Salari, Daniel Jost
}

\section{To cite this version:}

Maxime Mc Tortora, Hossein Salari, Daniel Jost. Chromosome dynamics during interphase: a biophysical perspective. Current Opinion in Genetics and Development, 2020, 61, pp.37-43. 10.1016/j.gde.2020.03.001 . hal-03012768

\section{HAL Id: hal-03012768 https://hal.science/hal-03012768}

Submitted on 18 Nov 2020

HAL is a multi-disciplinary open access archive for the deposit and dissemination of scientific research documents, whether they are published or not. The documents may come from teaching and research institutions in France or abroad, or from public or private research centers.
L'archive ouverte pluridisciplinaire HAL, est destinée au dépôt et à la diffusion de documents scientifiques de niveau recherche, publiés ou non, émanant des établissements d'enseignement et de recherche français ou étrangers, des laboratoires publics ou privés. 


\title{
Chromosome dynamics during interphase: a biophysical perspective
}

\author{
Maxime Tortora*, Hossein Salari", Daniel Jost ${ }^{+}$ \\ Université de Lyon, ENS de Lyon, Univ Claude Bernard, CNRS, Laboratory of Biology and Modeling \\ of the Cell, 46 Allée d'Italie, 69007 Lyon, France \\ * Contributed equally to this work \\ + Corresponding author; email: daniel.jost@ens-lyon.fr; phone: +33472728000
}

\section{Abstract}

The dynamic nature of chromosome organization plays a central role in the regulation of many crucial processes, such as DNA transcription and replication. However, the molecular bases of the link between genomic function, structure and dynamics remain elusive. In this review, we focus on how biophysical modelling can be instrumentally used to rationalize experimental studies of chromosome dynamics, and to probe the impact of putative mechanisms on genome folding kinetics during interphase. We introduce the general connection between chromatin internal organization and dynamics, and outline the potential effects of passive interactions mediated by architectural proteins and of active, energy-dependent processes on chromatin motion. Finally, we discuss current ambiguities emerging from in vivo observations, in particular related to ATP depletion and transcriptional activation, and highlight future perspectives.

\section{Keywords}

Chromatin motion, polymer modeling, chromosome organization, passive interactions, active processes

\section{Introduction}

Over the last decade, our understanding of chromosome organization and its relation to genome (mis-)function has dramatically improved, thanks to the advent of chromosome conformation capture $(\mathrm{Hi}-\mathrm{C})$ combined with rapid progress in genome engineering and microscopy [1]. Chromosomes are hierarchically organized at multiple scales: from the local packaging of nucleosomes into chromatin fiber that subsequently folds into sub-Mbp topologically-associating 
domains (TADs) to the spatial compartmentalization of genomic regions sharing the same epigenomic content and the large-scale positioning of chromosome territories.

Most current works are structural and focused on the average, 'static' 3D organization of the genome, along with its biological functions. However, many studies have highlighted the importance of chromosome dynamics [2]. Briefly, the live-tracking of fluorescently-tagged genomic loci or nucleosomes in living cells has evidenced the sub-diffusive, visco-elastic motion of the chromatin fiber (Fig.1A). Chromatin motion may strongly depend on transcriptional status [3-6], nuclear positioning [7-9] or DNA damage [10-13]. Whole genome dynamical studies have further revealed the existence of dynamically associated spatial regions, in which the motions of loci are correlated, and which closely match chromatin-associated nuclear compartments [5,14,15] (Fig.1B). Additionally, the use of 'static' methods like Hi-C at different time points also illustrates the global out-of-equilibrium dynamics of the genome, from the continuous decompaction of chromosomes after mitosis (Fig.1C) to their large-scale nuclear reorganization after hormonal induction [16] or DNA damage [17].

These observations suggest that a full understanding of the functional role of genomic spatial organization may only be achieved by accounting for its dynamical component. In particular, several mechanisms have been proposed for the regulation of the multi-scale 3D organization of chromosomes [18], from loop extrusion for TAD formation to micro-phase separation of chromatin-binding proteins for nuclear compartmentalization. However, very few studies have precisely quantified these dynamics, leaving a gap between the predicted outcomes of the processes and the experimental observations. Studying the dynamical properties of chromatin through the lens of biophysical modelling may thus provide a powerful tool to complement 'static' experiments, and help elucidate some of these underlying mechanisms.

\section{Generic impact of chromatin structure on chromosome dynamics}

Chromosomes consist of a polymeric chain, the chromatin fiber, which is usually modeled as a chain of elastic segments, and whose dynamics can be generically described by polymer physics concepts. In a crowded environment, as encountered in the nucleus, the local motion of genomic loci is mainly dictated by the elastic interactions exerted by neighboring consecutive segments. This is the so-called Rouse model [19], which is characterized by an anomalous sub-diffusive regime with $M S D(\tau)=\Gamma \tau^{\alpha}$ and $\alpha<1$ (Fig.1A), along with a visco-elastic behavior, in good agreement with many single-locus tracking studies in different species [3-5,20,21].

More generally, the dynamics of a polymer is intimately connected to its internal organization. Scaling arguments [22-25] provide a relation between the dynamic exponent $\alpha \simeq 2 \beta /(2 \beta+1)$ and the static Flory exponent $\beta$, which quantifies the increase of the average spatial distance $d(s) \sim s^{\beta}$ between two loci with their genomic separation $s$.

For instance, $\beta=1 / 2$ for short chains like yeast chromosomes [26], leading to the classical Rouse exponent $\alpha=1 / 2$ [19] (Fig.2A) observed for yeast chromatin in vivo [7] and in vitro [27]. Interestingly, in this regime, there exists a simple relation between the diffusion amplitude $\Gamma$ and the local properties of the polymer, such as its local rigidity [27] (Fig.2A). Socol et al [27] exploited 
this property to characterize the native yeast chromatin fiber using detailed Monte-Carlo simulations of nucleosome arrays. The chromatin parameters obtained from the comparison with in vitro MSD measurements of yeast-like chromatin (Kuhn length $b \sim 100 \mathrm{~nm}$; fiber compaction of $\sim 45 \mathrm{bp} / \mathrm{nm}$ ) were found to be consistent with the values inferred solely from structural data [28]. This relationship between fiber rigidity and dynamics was also invoked to interpret the increase of chromatin motion observed during DNA damage potentially due to changes in fiber properties around double-strand breaks [10-12].

For higher eukaryotes with longer chromosomes (Fig.2A), the generic organization is more compact $\beta=1 / 3$ [29]. This leads to $\alpha=0.4$, consistent with many experimental measurements obtained in mammalian cells [3-5,14,20-22] $(\alpha \sim 0.38-0.44)$ and with polymer simulations of genome folding $[30,31]$. This regime has been associated with the notions of crumpled polymers [32] (or fractal globules) that emerge from the decondensation of unknotted, mitotic-like polymers [29]. In this framework, the presence of topological constraints arising from steric interactions and confinement dramatically slows down the dynamics of large-scale organization. Genome folding thus remains out-of-equilibrium even at long timescales, as observed in $\mathrm{Hi}-\mathrm{C}$ or MSD studies on synchronized cells at different time points along the cell cycle [33,34] (Fig.1C).

\section{On the role of passive interactions in constraining chromatin motion}

The organization and dynamics of chromosomes in vivo are further impacted by the many mechanical forces that emerge from the various biochemical factors acting on chromatin. In particular, many processes driving small and large-scale genome organization (TAD formation, A/B compartmentalization, nuclear positioning) have been associated with 'passive', ATPindependent mechanisms involving protein-protein and/or protein-DNA interactions [18] (Fig. 2B). Polymer models have been instrumental in understanding the role of such interactions.

Generically, the presence of passive (transient or permanent) intra- or inter-chain loops constrains the local motion of monomers. This confinement effect inhibits their intrinsic ability to diffuse, leading to a decrease in the diffusion amplitude $\Gamma$, and may potentially induce a global compaction of the chromatin chain, leading to a smaller Flory exponent $\beta$, and thus a lower diffusion exponent $\alpha$ [35]. For example, Socol et al. [27] showed that a simple Rouse model decorated with nonspecific forces, putatively nucleosome-nucleosome interactions, may explain the difference in chromatin mobilities observed between in vivo and in vitro yeast chromatin $\left(\Gamma_{\text {vivo }} \sim \Gamma_{\text {vitro }} / 3\right)$ [27]. Interestingly, to fit both the experimental $\mathrm{Hi}-\mathrm{C}$ and MSD data, the lifetime of such transient loops in the model may exceed several seconds, considerably longer than typical timescales of chromatin local fluctuations $\left(\sim 10^{-2}-10^{-1} \mathrm{sec}\right.$. $)$ [30]. Loops may therefore play the role of momentary anchors for chromatin, perturbing dramatically its dynamics. In a different context, similar conclusions on loop lifetimes were inferred by Khanna et al when studying the constrained relative motion of $\mathrm{V}$ and DJ immunoglobulin segments in B-cells [20]. The MSD data was found to be consistent with a network of long-lived ( seconds) transient loops, typical of a gel phase near the sol-gel transition [20]. 
More specifically, the spatial partitioning of chromosomes into active/inactive (A/B) compartments emerges from the ability of architectural proteins like HP1 and PRC1 to oligomerize and/or form liquid-like condensates [36]. Polymer models [37] have suggested that such epigenetically-driven interactions may drive a (micro)-phase separation of chromatin states consistent with $\mathrm{Hi}-\mathrm{C}$ data and the formation of H3K9me3/HP1 intra-nuclear droplets [38]. The dynamical consequences of these interactions are numerous. Strongly-interacting regions will exhibit low mobility due to interactions with other monomers sharing the same chromatin state [30]. As heteroheterochromatin interactions were suggested to be stronger than their euchromatic counterparts based on polymer-physics interpretations of $\mathrm{Hi}-\mathrm{C}$ data $[37,39]$, this implies that heterochromatic loci may generally exhibit slower dynamics $[23,31,40]\left(\Gamma_{\text {hetero. }} \leq \Gamma_{\text {eu. }} ; \alpha_{\text {hetero. }} \leq \alpha_{\text {eu. }}\right)$, as observed experimentally [5,22]. Interestingly, polymer models also predict that monomers inside a given compartment move collectively over second-long periods [23,40], thus providing for a mechanistic interpretation of the micron-size dynamic compartments observed in mammalian nuclei $[5,14,15]$ (Fig.1B).

Passive interactions may also play an important role in the spatial positioning of the genome, notably at the nuclear periphery, although the microscopic origins of membrane-chromatin interactions remain unclear. The localization of genomic regions at the membrane leads to the reduction of their available diffusion space, and may hence result in smaller diffusion amplitudes $\Gamma$ for perinuclear loci $[7,41]$. MSD experiments have indeed shown a decrease in mobility of about $30-40 \%$ for regions preferentially interacting with the nuclear envelope like telomeres in yeast via SIR4 [7] or heterochromatin and lamina-associated domains in higher eukaryotes [22], potentially mediated by Lamin B1 [8]. Interestingly, another major component of the nuclear lamina, Lamin $A$, was shown to greatly influence chromosome dynamics throughout the nuclear interior $[42,43]$, suggesting that the presence of interactions between chromatin and the nucleoplasmic lamina network may help regulate gene mobility.

\section{Active processes enhance chromatin mobility}

Chromatin is also subjected to many energy-fueled processes, including nucleosome positioning, transcription, replication, loop extrusion or DNA repair. These active processes are intrinsically out-of-equilibrium and are expected to significantly affect the local and global dynamics of chromosomes. Although very few experiments have directly addressed the role of active processes on chromosome dynamics, many polymer models have been introduced to hypothesize their dynamical consequences.

At a coarse-grained level, an intuitive way of introducing the impact of active processes is via an increased, effective temperature acting on monomer diffusibility. "Hot", fast particles correspond to highly active, euchromatic regions while "cold", slow particles refer to more passive, heterochromatic loci. Polymer models accounting for such epigenetic-related heterogeneities in the local thermal fluctuations allowed to recapitulate structural effects like the A/B-type compartmentalization $[23,44]$. 
At a finer scale, active processes may be accounted for via an "active noise" that also enhances the local fluctuations of monomers. In this framework, genomic loci are considered as connected self-propelled particles moving in random directions [45], as connected extensile dipoles [46], or as passive monomers in an active viscous medium [47] (Fig. 2C). Theoretical analysis of such models has shown that the diffusivity of the polymer is significantly increased compared to standard Rouse model $\left(\Gamma_{\text {active }}>\Gamma_{\text {Rouse }}\right.$ ), and that the MSD may exhibit a super-Rousean regime $(\alpha>0.5)$ at short time-scales, while remaining Rousean or sub-Rousean $(\alpha \leq 0.5)$ at longer timescales. Interestingly, these predictions are consistent with experiments probing the dynamical effect of ATP where lower MSDs and weaker spatially-correlated motions were observed in yeast or mammalian cells following ATP depletion $[14,46,48]$. Furthermore, they provide a rationale for the in vivo observations of super-Rousean dynamics in various contexts $[3,42,43]$.

However, the exact nature of this active noise is still unclear. One potential contribution may arise from the intense traffic of active motors like chromatin remodeling factors or RNA and DNA polymerases on chromatin: according to Newton's third law, the directional displacement of molecular motors along the chromatin fiber may generate significant forces on the substrate [49]. For example, response to DNA damage is associated with an increased chromatin mobility that can be related to ATP-dependent processes [13], putatively via the motor activity of the ATPase Rad54 that can actively translocate along chromatin [50]. Another contribution may lie in nuclear myosin activity. Recent evidence in yeast suggests that nuclear myosins can bind to some transcription factors attached to chromatin, and hence propel the underlying genomic locus through the actin nuclear matrix, potentially over large (micron-scale) distances [51].

Some polymer models have also explicitly described the impact of specific active mechanisms on chromosome dynamics. Foglino et al. showed that the translocation of multiple fast motors (e.g., RNA polymerases) can exert large-enough mechanical forces on the chromatin fiber to enhance its overall mobility [49]. This is compatible with the higher diffusion amplitude $\Gamma$ observed by Gu et al. upon gene transcriptional activation in mouse ES and epiblast-like cells [4], but contradicts other experiments showing opposite effects in two human cell lines $[3,6]$. For the loop extrusion mechanism by SMCs involved in TAD formation and mitotic condensation [18], Nuebler et al. predicted that the passage of loop extruding factors will slightly increase the chromatin mobility, but only on a short time-scale representing the residence time of the loop extruding factor at a given locus [52]. However, this cannot explain the role of condensins in constraining local chromatin motion during mitosis as observed by MSD measurements in fission yeast [53].

Significant challenges nonetheless remain to fully characterize the role of ATP and energyconsuming processes in the regulation of chromosome dynamics. For example, global depletion of nuclear ATP has been associated with lower mobilities [14,46,48]. Is it mainly caused by a general reduction of molecular motor activity affecting the 'active noise' contribution of local fluctuations or by the expected increase of the nuclear concentration of $\mathrm{Mg}^{2+}$ cations normally chelated to ATP [54], that may lead to chromatin condensation [5,54], and thus to slower dynamics via passive (electrostatic) interactions [5]?

Similarly, how to interpret that inducing some active processes like transcription may inhibit chromatin mobility [3,6,14]? Clearly, an increase in active noise would enhance dynamics and 
cannot explain this phenomenon. Possibly, reduced dynamics may arise from passive interactions between active motors $[6,13]$ via the formation of transcription or replication factories, from the relocalization at the nuclear periphery [9] or from local changes in the chromatin fiber structure due to differential histone/epigenetic-marks turnover [10].

\section{Discussion}

Nuclear genome organization is driven by numerous passive and active processes that may have very different signatures on chromosome dynamics, but not necessarily on chromosome structure: the presence of passive interactions generally slows down the dynamics and constrains the motion of genomic loci, while active mechanisms are associated with an increase in chromatin mobility (Fig 2). The recent combination of structural and dynamical experiments with biophysical modeling has demonstrated the power of dynamical studies to assess quantitatively the basic mechanistic properties of chromatin. The future development of these approaches would be essential to rationalize novel experimental observations and disentangle current ambiguities. For example, they might be used to clarify whether the increased dynamics observed during DNA damage is due to a stiffening of the fiber [12], to local chromatin decompaction, which might result from changes in histone degradation [10,11], and/or to the higher activity of some molecular motors [13] like Rad54 trafficking on chromatin.

In this context, probing chromosome dynamics using biophysical models may provide a significant contribution to our understanding of its functional roles, and may shed new light on how chromatin fiber properties, passive and active forces impact the dynamical communication between distal loci. These considerations are especially relevant for promoter-enhancer interactions [55]. Indeed, the molecular mechanisms behind the transcriptional control by enhancers remain largely unknown. Current views propose an activity-by-contact model where activation is related to spatial proximity $[56,57]$. The analysis of the dynamical aspects of such models by studying the firstencounter and residence times between promoters and enhancers $[30,58]$ could provide one of the keys to gain new insights into this crucial regulatory process.

Finally, the link between nuclear ATP, active processes and chromatin mobility would deserve further scrutiny. The direct correlation between nuclear ATP levels and ionic content $[5,54]$ suggests that ATP depletion experiments alone are not sufficient to disentangle the respective roles of electrostatic condensation and of ATP-dependent activity in the observed alterations of chromatin dynamics [14,46,48]. Further experimental studies based on the precise jointmeasurement of nuclear ATP and free $\mathrm{Mg}^{2+}$ concentrations [54] as well as on the systematic inhibition of chromatin-binding ATPases [5] would be highly desirable to resolve these important ambiguities. Similarly, perturbation experiments decoupling the motor activity of key complexes like RNA polymerases from their intrinsic capacity to self-interact or phase separate [59] would allow for a rigorous assessment of the relevance of the different proposed mechanisms on chromatin activity. 


\section{Acknowledgments}

We apologize to colleagues whose work could not be cited due to space constraints. We thank Guillermo Orsi, Jean-Michel Arbona, Cédric Vaillant and Marco Di Stefano for critical reading of the manuscript. We acknowledge Agence Nationale de la Recherche (ANR-18-CE12-0006-03, ANR-18-CE45-0022-01) for funding.

The authors declare no conflict of interest.

\section{Figure legends}

Figure 1: Experimental observations on chromosome dynamics. (A) (Top) Dynamics of chromosome loci are mainly investigated by tracking in live cells the motion of fluorescentlytagged single loci [2] over time ( from seconds to minutes) in 2D optical layers. (Down) Statistical quantification is usually performed by computing the temporal variation of the mean squared displacement (MSD) which represents the average displacement after a time lag $\tau: M S D(\tau)=<$ $(\boldsymbol{r}(t+\tau)-r(t))^{2}>$ with $r(t)$ the position vector and $<.>$ the time-average over one trajectory or the ensemble-average over the many trajectories. Typically, the MSD depends on $\tau$ following a power-law: $M S D(\tau)=\Gamma \tau^{\alpha}$ with $\alpha$ the diffusion exponent and $\Gamma$ the amplitude. For example, for a particle freely diffusing in three dimensions, $\alpha=1$ and $\Gamma=6 D$ with $D$ the diffusion coefficient. Most of the MSD measurement for chromatin illustrates the sub-diffusivity of genomic motion with $\alpha \sim 0.4-0.5$ and $\Gamma$ that may depend on the cell type or transcriptional status. Data were extracted from $[3,4,7,21]$. (B) (Top) Large-scale, genome-wide information on chromosome dynamics may also be studied by following the minutes-long spatio-temporal evolution of the intensity of stained histones in live cells $[5,14,15]$. (Down) Time-resolved image correlation analysis during a time interval $\Delta t$ revealed the existence of micron-scale dynamically-associated compartments whose loci have coherent movement during $\Delta t$ of a few seconds. Schemes were inspired from results in [14]. (C) (Top) Analysis of the slow, large-scale dynamics of genome organization is studied with $\mathrm{Hi}-\mathrm{C}$ experiments on synchronized cells along the cell cycle [33,34]. After mitosis, the decompaction of chromosomes is gradual: sub-Mbp organization converging rapidly to steadystate while large-scale contacts and compartmentalization remaining out-of-equilibrium. (Down) This can be quantified by estimating the average contact frequency between two loci as a function of their genomic distance at different time points of the cell cycle after mitotic release, that exhibits different dynamics at intermediate or large scales. Data were extracted from [33].

Figure 2: Impact of polymer properties, passive and active forces on chromatin motion. (A) Effects of local polymer structure and internal organization on chain diffusion. (Top) In the regime of limited chromatin compaction, as in the case of yeast chromosomes, the dynamics of the chain may be well described by the Rouse model, $M S D(\tau)=\Gamma_{\text {Rouse }} \tau^{0.5}$. The diffusion amplitude $\Gamma_{\text {Rouse }} \sim$ $b / \sqrt{\zeta}$ may then be related to the monomer friction coefficient $\zeta$, which generally depends on the local chain geometry, and to the so-called Kuhn length $b$, defined as the genomic separation distance beyond which the orientations of two distinct chain segments are statistically 
uncorrelated [27]. Increasing chromatin rigidity may thus enhance the polymer dynamics in diluted systems, albeit without affecting the long-time diffusion exponent $\alpha$ obtained from the slope of the MSD curve in log-log scale. (Bottom) In higher eukaryotes, the denser organization of chromosomes is generally better captured by the slower crumpled polymer dynamics, $M S D(\tau)=$ $\Gamma_{\text {crump }} \tau^{0.4}$, with $\Gamma_{\text {crump }}<\Gamma_{\text {Rouse }}$. (B) Schematic representation of a passive polymer with specific attractive interactions. In this model, blue and green monomers may respectively bind to one another, and black particles may bind to the nuclear membrane, further constraining the chain dynamics. Orange monomers are assumed to be inert. Passive forces usually lead to slower dynamics ( $\Gamma \leq \Gamma_{\text {Rouse }} ; \alpha \leq \alpha_{\text {Rouse }}$ ). (C) (Left) Schematic representation of an active polymer. The chain consists of connected, self-propelled monomers known as active Brownian particles, whose velocities bear a constant magnitude and a randomly-diffusive direction (black arrows). Polar active polymers correspond to the case in which the monomer velocity vector is always borne by the local tangent to the chain backbone. (Right) Schematic representation of translocating motors acting on chromatin. In this model, molecular motors (blue triangles) diffuse in 3D space and may bind/unbind to a passive polymer (orange curve). Actives forces usually lead to faster dynamics ( $\Gamma \geq \Gamma_{\text {Rouse }} ; \alpha \geq \alpha_{\text {Rouse }}$ ).

\section{References}

1. Rowley MJ, Corces VG. Organizational principles of 3D genome architecture. Nat Rev Genet. 2018;19: 789-800.

2. Bystricky K. Chromosome dynamics and folding in eukaryotes: Insights from live cell microscopy. FEBS Lett. 2015;589: 3014-3022.

3. Germier T, Kocanova S, Walther N, Bancaud A, Shaban HA, Sellou H, et al. Real-Time Imaging of a Single Gene Reveals Transcription-Initiated Local Confinement. Biophys J. 2017;113: 1383-1394.

4. Gu B, Swigut T, Spencley A, Bauer MR, Chung M, Meyer T, et al. Transcription-coupled changes in nuclear mobility of mammalian cis-regulatory elements. Science. 2018;359: 1050-1055.

5. Nozaki T, Imai R, Tanbo M, Nagashima R, Tamura S, Tani T, et al. Dynamic Organization of Chromatin Domains Revealed by Super-Resolution Live-Cell Imaging. Mol Cell. 2017;67: 282-293.e7.

6. Nagashima R, Hibino K, Ashwin SS, Babokhov M, Fujishiro S, Imai R, et al. Single nucleosome imaging reveals loose genome chromatin networks via active RNA polymerase II. J Cell Biol. 2019;218: 1511-1530.

7. Hajjoul H, Mathon J, Ranchon H, Goiffon I, Mozziconacci J, Albert B, et al. High-throughput chromatin motion tracking in living yeast reveals the flexibility of the fiber throughout the genome. Genome Res. 2013;23: 1829-1838.

8. Chang L, Li M, Shao S, Xue B, Hou Y, Zhang Y, et al. Chromatin-lamin B1 interaction promotes genomic compartmentalization and constrains chromatin dynamics. 
doi:10.1101/601849

9. Zhang H, Petrie MV, He Y, Peace JM, Chiolo IE, Aparicio OM. Dynamic relocalization of replication origins by Fkh1 requires execution of DDK function and Cdc45 loading at origins. Elife. 2019;8. doi:10.7554/eLife.45512

10. Hauer $M H$, Seeber A, Singh V, Thierry R, Sack R, Amitai A, et al. Histone degradation in response to DNA damage enhances chromatin dynamics and recombination rates. Nat Struct Mol Biol. 2017;24: 99-107.

** This study (in combination with Ref. [11]) shows that DNA damage leads to massive histone eviction. Proposed polymer modeling and experiments suggest that this loss triggers changes in the local chromatin structure that may explain the enhancement of chromatin mobility after DNA breaks.

11. Amitai A, Seeber A, Gasser SM, Holcman D. Visualization of Chromatin Decompaction and Break Site Extrusion as Predicted by Statistical Polymer Modeling of Single-Locus Trajectories. Cell Rep. 2017;18: 1200-1214.

12. Herbert S, Brion A, Arbona J-M, Lelek M, Veillet A, Lelandais B, et al. Chromatin stiffening underlies enhanced locus mobility after DNA damage in budding yeast. EMBO J. 2017;36: 2595-2608.

13. Eaton JA, Zidovska A. Structural and Dynamical Signatures of Local DNA Damage in Live Cells. Biophys J. 2019. doi:10.1016/j.bpj.2019.10.042

14. Zidovska A, Weitz DA, Mitchison TJ. Micron-scale coherence in interphase chromatin dynamics. Proc Natl Acad Sci U S A. 2013;110: 15555-15560.

15. Shaban HA, Barth R, Bystricky K. Formation of correlated chromatin domains at nanoscale dynamic resolution during transcription. Nucleic Acids Res. 2018;46: e77.

16. Zhou Y, Gerrard DL, Wang J, Li T, Yang Y, Fritz AJ, et al. Temporal dynamic reorganization of $3 \mathrm{D}$ chromatin architecture in hormone-induced breast cancer and endocrine resistance. Nat Commun. 2019;10: 1522.

17. Aymard F, Aguirrebengoa M, Guillou E, Javierre BM, Bugler B, Arnould C, et al. Genomewide mapping of long-range contacts unveils clustering of DNA double-strand breaks at damaged active genes. Nat Struct Mol Biol. 2017;24: 353-361.

18. Mirny LA, Imakaev M, Abdennur N. Two major mechanisms of chromosome organization. Curr Opin Cell Biol. 2019;58: 142-152.

19. Doi M, Edwards SF. The Theory of Polymer Dynamics. Oxford University Press; 1988.

20. Khanna N, Zhang Y, Lucas JS, Dudko OK, Murre C. Chromosome dynamics near the solgel phase transition dictate the timing of remote genomic interactions. Nat Commun. 2019;10: 2771.

** In this study, locus tracking experiments are combined with a hierarchy of polymer models to investigate the dynamics of V-DJ recombination in B-lymphocytes. It is found that the constrained motion of chromatin observed in vivo is consistent with that of a network of long-lived intra-chain loops near the sol-gel transition, thus 
providing for an optimal tradeoff between order and fluidity to facilitate genomic function.

21. Chen B, Gilbert LA, Cimini BA, Schnitzbauer J, Zhang W, Li G-W, et al. Dynamic imaging of genomic loci in living human cells by an optimized CRISPR/Cas system. Cell. 2013;155: 1479-1491.

22. Shinkai S, Nozaki T, Maeshima K, Togashi Y. Dynamic Nucleosome Movement Provides Structural Information of Topological Chromatin Domains in Living Human Cells. PLoS Comput Biol. 2016;12: e1005136.

23. Liu L, Shi G, Thirumalai D, Hyeon C. Chain organization of human interphase chromosome determines the spatiotemporal dynamics of chromatin loci. PLOS Computational Biology. 2018. p. e1006617. doi:10.1371/journal.pcbi.1006617

24. Amitai A, Holcman D. Polymer physics of nuclear organization and function. Phys Rep. 2018; 678: 1-83.

25. Polovnikov KE, Gherardi M, Cosentino-Lagomarsino M, Tamm MV. Fractal Folding and Medium Viscoelasticity Contribute Jointly to Chromosome Dynamics. Phys Rev Lett. 2018;120: 088101.

26. Kimura H, Shimooka Y, Nishikawa J-I, Miura O, Sugiyama S, Yamada S, et al. The genome folding mechanism in yeast. J Biochem. 2013;154: 137-147.

27. Socol M, Wang R, Jost $D$, Carrivain $P$, Vaillant $C$, Le Cam E, et al. Rouse model with transient intramolecular contacts on a timescale of seconds recapitulates folding and fluctuation of yeast chromosomes. Nucleic Acids Res. 2019;47: 6195-6207.

** This study combines dynamical tracking experiments and biophysical modeling to infer physico-chemical properties of chromatin fiber. The authors use in vitro MSD measurements on reconstituted chromatin to infer its mechanical properties and show that the in vivo dynamics is consistent with a Rouse model with transient molecular interactions on a timescale of seconds.

28. Arbona J-M, Herbert S, Fabre E, Zimmer C. Inferring the physical properties of yeast chromatin through Bayesian analysis of whole nucleus simulations. Genome Biol. 2017;18: 81.

29. Rosa A, Everaers R. Structure and dynamics of interphase chromosomes. PLoS Comput Biol. 2008;4: e1000153.

30. Ghosh SK, Jost D. How epigenome drives chromatin folding and dynamics, insights from efficient coarse-grained models of chromosomes. PLoS Comput Biol. 2018;14: e1006159.

* In this study, coarse-grained polymer models are developed to describe out-ofequilibrium chromosome organization and dynamics. Applications to drosophila illustrate how passive, epigenetically-driven interactions may slow down local chromatin motion and may participate in the regulation of communications between distal loci.

31. Shi G, Liu L, Hyeon C, Thirumalai D. Interphase human chromosome exhibits out of 
equilibrium glassy dynamics. Nature Communications. 2018. doi:10.1038/s41467-01805606-6

32. Halverson JD, Smrek J, Kremer K, Grosberg AY. From a melt of rings to chromosome territories: the role of topological constraints in genome folding. Rep Prog Phys. 2014;77: 022601.

33. Abramo K, Valton A-L, Venev SV, Ozadam H, Fox AN, Dekker J. A chromosome folding intermediate at the condensin-to-cohesin transition during telophase. Nat Cell Biol. 2019;21: 1393-1402.

34. Ma H, Tu L-C, Chung Y-C, Naseri A, Grunwald D, Zhang S, et al. Cell cycle- and genomic distance-dependent dynamics of a discrete chromosomal region. The Journal of Cell Biology. 2019. pp. 1467-1477. doi:10.1083/jcb.201807162

35. Bohn M, Heermann DW. Diffusion-driven looping provides a consistent framework for chromatin organization. PLoS One. 2010;5: e12218.

36. Mir M, Bickmore W, Furlong EEM, Narlikar G. Chromatin topology, condensates and gene regulation: shifting paradigms or just a phase? Development. 2019. p. dev182766.

doi:10.1242/dev.182766

37. Vaillant $C$, Jost $D$. Modeling the Functional Coupling between 3D Chromatin Organization and Epigenome. Modeling the 3D Conformation of Genomes. 2019. pp. 21-56. doi:10.1201/9781315144009-2

38. Wang L, Gao Y, Zheng X, Liu C, Dong S, Li R, et al. Histone Modifications Regulate Chromatin Compartmentalization by Contributing to a Phase Separation Mechanism. Mol Cell. 2019;76: 646-659.e6.

39. Falk M, Feodorova Y, Naumova N, Imakaev M, Lajoie BR, Leonhardt H, et al. Publisher Correction: Heterochromatin drives compartmentalization of inverted and conventional nuclei. Nature. 2019;572: E22.

40. Di Pierro M, Potoyan DA, Wolynes PG, Onuchic JN. Anomalous diffusion, spatial coherence, and viscoelasticity from the energy landscape of human chromosomes. Proc Natl Acad Sci U S A. 2018;115: 7753-7758.

* This paper introduces a polymer model that accounts for epigenetically-driven, passive interactions and whose parameters were fitted to predict the 3D 'static' organization of the human genome. The authors show that such a model correctly reproduces the spatial coherence, visco-elastic behavior and subdiffusive regime observed experimentally for chromosome dynamics.

41. Amitai A, Toulouze M, Dubrana K, Holcman D. Analysis of Single Locus Trajectories for Extracting In Vivo Chromatin Tethering Interactions. PLoS Comput Biol. 2015;11: e1004433.

42. Bronshtein I, Kepten E, Kanter I, Berezin S, Lindner M, Redwood AB, et al. Loss of lamin A function increases chromatin dynamics in the nuclear interior. Nature Communications. 2015. doi:10.1038/ncomms9044 
43. Taheri F, Isbilir B, Müller G, Krieger JW, Chirico G, Langowski J, et al. Random Motion of Chromatin Is Influenced by Lamin A Interconnections. Biophysical Journal. 2018. pp. 24652472. doi:10.1016/j.bpj.2018.04.037

44. Agrawal A, Ganai N, Sengupta S, Menon GI. Nonequilibrium Biophysical Processes Influence the Large-Scale Architecture of the Cell Nucleus. Biophys J. 2019. doi:10.1016/j.bpj.2019.11.017

45. Mousavi SM, Mahdiyeh Mousavi S, Gompper G, Winkler RG. Active Brownian ring polymers. The Journal of Chemical Physics. 2019. p. 064913. doi:10.1063/1.5082723

46. Saintillan D, Shelley MJ, Zidovska A. Extensile motor activity drives coherent motions in a model of interphase chromatin. Proc Natl Acad Sci U S A. 2018;115: 11442-11447.

* This paper proposes to model chromatin as a polymer acted upon by extensible dipolar active motors, which account for energy-dependent enzymatic activity. The authors show how dynamically-associated compartments emerge naturally from this system and how ATP-depletion may impact the coherence and dynamics of these compartments.

47. Chaki S, Chakrabarti R. Enhanced diffusion, swelling, and slow reconfiguration of a single chain in non-Gaussian active bath. J Chem Phys. 2019;150: 094902.

48. Weber SC, Spakowitz AJ, Theriot JA. Nonthermal ATP-dependent fluctuations contribute to the in vivo motion of chromosomal loci. Proceedings of the National Academy of Sciences. 2012. pp. 7338-7343. doi:10.1073/pnas.1119505109

49. Foglino M, Locatelli E, Brackley CA, Michieletto D, Likos CN, Marenduzzo D. Nonequilibrium effects of molecular motors on polymers. Soft Matter. 2019;15: 5995-6005.

50. Mazin AV, Mazina OM, Bugreev DV, Rossi MJ. Rad54, the motor of homologous recombination. DNA Repair . 2010;9: 286-302.

51. Wang A, Kolhe JA, Gioacchini N, Baade I, Brieher WM, Peterson CL, et al. Mechanism of Long-Range Chromosome Motion Triggered by Gene Activation. Dev Cell. 2019. doi:10.1016/j.devcel.2019.12.007

52. Nuebler J, Fudenberg G, Imakaev M, Abdennur N, Mirny LA. Chromatin organization by an interplay of loop extrusion and compartmental segregation. Proc Natl Acad Sci U S A. 2018;115: E6697-E6706.

53. Kakui Y, Rabinowitz A, Barry DJ, Uhlmann F. Condensin-mediated remodeling of the mitotic chromatin landscape in fission yeast. Nat Genet. 2017;49: 1553-1557.

54. Maeshima K, Matsuda T, Shindo Y, Imamura H, Tamura S, Imai R, et al. A Transient Rise in Free Mg2 lons Released from ATP-Mg Hydrolysis Contributes to Mitotic Chromosome Condensation. Current Biology. 2018. pp. 444-451.e6. doi:10.1016/j.cub.2017.12.035

55. van Steensel B, Furlong EEM. The role of transcription in shaping the spatial organization of the genome. Nat Rev Mol Cell Biol. 2019;20: 327-337.

56. Fulco CP, Nasser J, Jones TR, Munson G, Bergman DT, Subramanian V, et al. Activity-bycontact model of enhancer-promoter regulation from thousands of CRISPR perturbations. 
Nature Genetics. 2019. pp. 1664-1669. doi:10.1038/s41588-019-0538-0

57. Chen H, Levo M, Barinov L, Fujioka M, Jaynes JB, Gregor T. Dynamic interplay between enhancer-promoter topology and gene activity. Nature Genetics. 2018. pp. 1296-1303. doi:10.1038/s41588-018-0175-z

58. Doyle B, Fudenberg G, Imakaev M, Mirny LA. Chromatin loops as allosteric modulators of enhancer-promoter interactions. PLoS Comput Biol. 2014;10: e1003867.

59. Lu H, Yu D, Hansen AS, Ganguly S, Liu R, Heckert A, et al. Phase-separation mechanism for C-terminal hyperphosphorylation of RNA polymerase II. Nature. 2018;558: 318-323. 